\title{
Kinetics of phase transitions in weakly segregated block copolymers: Pseudostable and transient states
}

\author{
Shuyan Qi \\ Division of Physics, Mathematics and Astronomy, California Institute of Technology, Pasadena, California 91125 \\ Zhen-Gang Wang ${ }^{*}$ \\ Division of Chemistry and Chemical Engineering, California Institute of Technology, Pasadena, California 91125
}

(Received 2 August 1996)

\begin{abstract}
We study the kinetics of order-disorder and order-order transitions in weakly segregated diblock copolymers using a time-dependent Ginzburg-Landau (TDGL) approach. In particular, we investigate the microstructural change as well as the order-parameter evolution after a sudden temperature jump from one phase to another. Direct numerical simulation of the TDGL equations shows that depending on the extent of the temperature jump, these transitions often occur in several stages and can involve nontrivial intermediate states. For example, we find that transition from the lamellar phase to the hexagonal cylinder phase goes through a perforated lamellar state within a certain temperature range. The numerical results are elucidated by a multimode analysis under the single-wave-number approximation. The analysis reveals that the geometric characteristics of the free energy surface, particularly saddle points and ridgelike features, are responsible for the nontrivial intermediate states on the kinetic pathways. On the basis of this analysis, a generalized kinetic "phase diagram" is constructed, which is able to account for all the different scenarios observed in the numerical simulation. Our results are discussed in connection with available experimental observations. In particular, we suggest the possibility that the perforated-modulated lamellar structures obtained by Bates and co-workers [I. W. Hamley, K. A. Koppi, J. H. Rosedale, F. S. Bates, K. Almdal, and K. Mortensen, Macromolecules 26, 5959 (1993); S. Förster, A. K. Khandpur, J. Zhao, F. S. Bates, I. W. Hamley, A. J. Ryan, and W. Bras, Macromolecules 27, 6922 (1994)] may be kinetic, intermediate states rather than new equilibrium phases. [S1063-651X(97)10301-4]

PACS number(s): 64.70.-p, 81.30.Hd, 83.70.Hq
\end{abstract}

\section{INTRODUCTION}

One of the most fascinating properties of block copolymers is their ability to self-assemble into a variety of ordered microstructures. The simplest block copolymer system is an undiluted diblock copolymer with two incompatible blocks, where upon decreasing the temperature and/or increasing the molecular weight, a variety of ordered microstructures, such as body-center-cubic (BCC) spheres, hexagonally ordered cylinders (HEX), lamellae (LAM), and more complex bicontinuous structures have been obtained [1]. The equilibrium morphological behaviors of such simple diblock copolymers are now well understood. Relevant to the current work is that fact that in the weak segregation limit (i.e., near the orderdisorder transition), phase transitions can be effected by changing the temperature. Thus an interesting and natural question is what are the kinetic pathways during the various order-order and order-disorder transitions after a sudden temperature change? More specifically, how does the system transform from one microstructure to another (of different symmetry)? Are there interesting intermediate states during the transition? If so, what is the nature of these intermediate states?

In this paper, we address these questions by a time-

*Author to whom correspondence should be addressed. dependent Ginzburg-Landau (TDGL) approach. Apart from the fundamental interest in understanding the kinetics of phase transitions involving spatially modulated phases, answers to these questions are also helpful in designing suitable processing routes for obtaining well-ordered structures in self-assembling systems. For example, our preliminary results [2] indicate that hexagonal cylinders obtained by a temperature jump from a defect-free lamellar structure do not contain many structural defects, whereas the same structure obtained by a temperature quench from the disordered or even the BCC phase will usually be laden with defects. This in turn raises an interesting question about the role of the initial symmetry in directing the structural evolution of another phase.

To be concrete, we shall use $A-B$ diblock copolymers as the context, although the same analysis can be easily extended to other systems. For diblock copolymers, the appropriate order parameter is $\psi(\vec{r})=2\left(f-\phi_{A}(\vec{r})\right)$ $=\phi_{B}(\vec{r})-\phi_{A}(\vec{r})-(1-2 f)$, where $\phi_{A}(\vec{r})$ and $\phi_{B}(\vec{r})$ are, respectively, the local volume fractions of $A$ and $B$ monomers, and $f$ is the fraction of $A$ monomers in the diblock. The time evolution of the order parameter $\psi$ is governed, in the spirit of linear irreversible thermodynamics, by the following timedependent Ginzburg-Landau equation [3]:

$$
\frac{\partial \psi}{\partial t}=M \nabla^{2}\left(\frac{\delta F}{\delta \psi}\right)+\eta(\vec{r}, t),
$$


where we have used the fact that $\psi$ is a conserved order parameter as is appropriate for diblock copolymers. In Eq. (1), $M$ is a mobility coefficient, which we assume to be a constant; $\eta(\vec{r}, t)$ is a random force, which for a system in equilibrium at temperature $T$, satisfies the fluctuationdissipation relation

$$
\left\langle\eta(\vec{r}, t) \eta\left(\overrightarrow{r^{\prime}}, t^{\prime}\right)\right\rangle=-2 M k_{B} T \nabla^{2} \delta\left(\vec{r}-\vec{r}^{\prime}\right) \delta\left(t-t^{\prime}\right) ;
$$

and $F[\psi(\vec{r})]$ is the free energy functional which we take to be of the form

$$
\begin{aligned}
F[\psi(\vec{r})]= & \int d \vec{r}\left\{\psi(\vec{r})\left[-\frac{\tau}{2}+\frac{a}{2}(1-2 f)^{2}-\frac{b}{2} \nabla^{2}\right] \psi(\vec{r})\right. \\
& \left.+\frac{v}{3}(1-2 f) \psi(\vec{r})^{3}+\frac{u}{4} \psi(\vec{r})^{4}\right\} \\
& +\frac{c}{2} \int d \overrightarrow{r_{1}} \int d \overrightarrow{r_{2}} G\left(\overrightarrow{r_{1}}-\overrightarrow{r_{2}}\right) \psi\left(\overrightarrow{r_{1}}\right) \psi\left(\overrightarrow{r_{2}}\right) .
\end{aligned}
$$

This form of the free energy can be considered as an expansion in the order parameter $\psi$ and $1-2 f$. The long-range interaction in the last intergral arises from the connectivity of the two blocks and is typical of amphiphilic systems [4-6], where $G\left(\overrightarrow{r_{1}}-\overrightarrow{r_{2}}\right)$ satisfies $\nabla^{2} G\left(\overrightarrow{r_{1}}-\overrightarrow{r_{2}}\right)=-\delta\left(\overrightarrow{r_{1}}-\overrightarrow{r_{2}}\right) \cdot \tau$ is related to the order-disorder transition temperature and the coefficients $a, b, c, u$, and $v$ can be computed by evaluating the appropriate vertex functions given by Leibler [7] together with the local approximation for the higher (third and fourth) vertex functions $[5,8]$. We point out that a large class of physical systems with competing short-range and long-range interactions can be described by a free energy functional of the type in Eq. (3) [9]. Thus we believe that the phenomenology we are studying is quite general and, hence, we allow the freedom of choosing the parameters in Eq. (3) as phenomenological constants not specifically limited to diblock copolymers.

Derivation of the TDGL equations from the microscopic chain dynamics generally yield a nonlocal mobility coefficient [10-12]. Here we ignore this nonlocality, by assuming a constant mobility $M$. This should not be a serious approximation in the weak-segregation regime since the order parameter in this regime is dominated by a single wave number. By the same token, the distinction between conserved and nonconserved order-parameter dynamics is not essential, and, therefore, most of our results for the case of conserved order parameters should be applicable also for nonconserved order parameters.

Several dynamical aspects of systems that can be described by the free energy funtional Eq. (3) have been addressed in the literature. Desai and co-workers, using a combination of numerical and analytical approaches, have extensively investigated the kinetics of microstructure formation from the disordered phase [13]. Oono and co-workers $[14,15]$ developed a cell-dynamics approach for simulating the formation of microstructures in diblock copolymers. Chakrabarti, Toral, and Gunton using the cell-dynamics approach combined with simulated annealing, showed that large, defect-free lamellar domains can be obtained with a sufficiently slow cooling rate [16]. A number of researchers have addressed the rheological properties of block copolymers near the order-disorder transition $[17,18]$ and in the ordered phases $[19,20]$. In recent years, flow-induced ordering and alignment of microstructures by flow in block copolymers, have attracted much attention both theoretically [21-24] and experimentally [25]. While these dynamical issues are all important and interesting, we feel that the simpler but no less important question of the kinetics of transitions between different ordered phases and between an ordered and the disordered phases in the quiescent condition need to be addressed. The kinetics turn out to be far from trivial, even in transitions where there is no free energy barrier: as we will demonstrate in this paper, pseudostable and transient states on the kinetic pathway, which are due, respectively, to saddle and ridgelike features on the free energy surface, give rise to interesting intermediate structures during the transition and to nonsimple temporal behaviors. Some results for the hexagonal to BCC and hexagonal to disordered phase transitions were reported by us recently in a brief communication [26]. The present paper is a more extended study that includes other transitions and also encompasses a broader range of issues.

The TDGL equation will be analyzed by two methods: direct numerical simulation and a simplified mode analysis using the single-wave-number approximation. The temperature change is mimicked by a change in the parameter $\tau$ from an initial value favoring one phase $\tau_{i}$ to a value favoring a different phase $\tau_{f}$. Broadly speaking, an increase in $\tau$ corresponds to a decrease in temperature and vice versa. In this work we focus on temperature-jump ( $T$ jump) kinetics because in temperature-quench cases, the kinetics usually involve the formation of complicated topological defects accompanying the lowering of symmetry in a system. Inclusion of such defects requires a much larger system in the direct numerical simulation and more sophisticated analytical approaches than employed here. Also, we avoid the issue of nucleation and consider cases where the $T$ jumps are beyond the spinodal of the initial phase in question. Thus the transitions we study are barrierless and the microstructural changes are global. Finally, we limit our study to transitions involving the lamellar (LAM), hexagonal cylinder (HEX), body-center-cubic (BCC), and disordered (DIS) phases. We omit the gyroid phase [27] since this phase is not stable in the immediate vicinity of the order-disorder transition $[28,29]$.

This paper is organized as follows. In Sec. II, results from direct numerical simulation of the TDGL equation are presented for several order-order and order-disorder transitions. The temporal evolution of the system is monitored by defining a global order parameter to indicate the different stages of the kinetic pathways. Three-dimensional gray-level plots are used to show more directly the microstructural evolution. These numerical results are elucidated in Sec. III by a multimode analysis under a single-wave-number approximation. We highlight two types of intermediate states: the pseudostable state [30] and the transient state which correspond, respectively, to saddle and ridgelike features in the free energy surface. A generalized phase diagram which include the various metastability and pseudostability limits as well as regions in which other interesting intermediate structures are to be observed, is constructed. Our results are discussed in 
Sec. IV in connection with available experiments and also in the context of some broader issues in the behavior of block copolymer mesophases.

\section{DIRECT NUMERICAL SIMULATION}

In this section, we present results from direct numerical simulation of Eq. (1). The parameters are choosen to be $u=0.5, v=1.5, \quad b=0.5, \quad c=0.02$, and $a=1.5$. To facilitate the numerical procedure, we bring Eq. (1) into a celldynamics form suggested by Oono and co-workers $[14,15]$. The numerical simulations are performed on a $48 \times 48 \times 50$ simple cubic lattice. An initial ordered structure (LAM, HEX, BCC) is prepared at the initial temperature $\tau_{i}$; then we change $\tau$ to $\tau_{f}$ and follow the subsequent evolution of the system after this sudden change in temperature. To be consistent with the mean-field level mode analysis in Sec. III, we will ignore the thermal noise term in Eq. (1), except to include it as providing an initial randomness (for possible symmetry breaking). We have conducted studies that include the fluctuation during the simulation and do not find its effects to be essential. The effects of fluctuations will be discussed further in Sec. IV.

Two different procedures are used to generate the initial defect-free ordered phases. One is to set a sinusoidal wave with the appropriate symmetry of the state (with the wavelength determined from the free energy) and then let the system equilibriate for a suffciently long time. Another procedure is to let the ordered phase evolve from the disordered state by adding a random but anisotropic initial perturbation determined by the symmetry of the state. The two schemes lead to the same ordered structures. In both cases, we make sure that the initial ordered phase is the equilibrium structure at the temperature $(\tau)$ from the mean-field diagram.

To quantify the global temporal behavior of the system, we introduce an order parameter $Q=\sqrt{\sum_{\text {sites }} \psi(\vec{r})^{2}}$ $=\sqrt{\sum_{k} \psi_{k} \psi_{-k}}$. At the mean-field level, $Q$ is zero in the disordered phase and is positive in the ordered phases. The time evolution of $Q$ is used to demonstrate different stages in the kinetics. Where there involve interesting intermediate states, we also show three-dimensional gray-level plots of the order parameters or two-dimensional cross sections. The different transitions are listed below. Cross references are made between this section and Sec. III in order to corroborate between the numerical findings and results from the simplified mode analyses.

\section{A. LAM to HEX transition}

A transition from LAM to the hexagonal cylinder (HEX) phase is possible only for asymmetric diblocks. Here we choose $f=0.45$. The initial value of the temperaturelike parameter $\tau_{i}=0.35$. The mean-field equilibrium structure corresponding to this set of parameters is the lamellar structure. The structure is shown in Fig. 1(a), with the layer normal along the $z$ direction. Three temperature jumps are studied with the final $\tau_{f}$ equal $0.25,0.23$, and 0.22 , respectively, all within the HEX phase in the mean-field phase diagram. On physical grounds, we expect the cylinders to pinch off from the lamellar layers containing the minority blocks, with their axes lying within the initial lamellar layers.
Figure 2 shows the temporal evolution of the global order parameter $Q$. For all three temperature jumps, the order parameter reaches a final steady-state value consistent with the equilibrium HEX phase. However, in all three cases, the evolution is nonmonotonic. In the cases of the two lower temperatures (or larger $\tau_{f}$ values), there is a clear plateau following the initial rapid decrease in the order parameter, suggesting that the system reaches some kind of stagnation point. The duration of the plateau is clearly longer for the lower temperature $\left(\tau_{f}=0.25\right)$ than for the higher temperature $\left(\tau_{f}=0.23\right)$. For $\tau_{f}=0.22$ the plateau is less pronounced.

Direct visual inspection of the gray-level plot of the microstructures during the first plateau region does not reveal any specific structure within the lamellar layers. Thus the system remains in the lamellar phase, with a decreased order parameter. A multimode analysis using the single wavenumber approximation in Sec. III shows that the first plateau in Fig. 2 corresponds to a saddle-point in the free energy surface.

Of special interest is the appearance of a second plateau in the two lower temperature cases. A gray-level orderparameter plot [Fig. 1(b)] shows that at the end of the first plateau, modulation within the lamellar layers begins to develop. The structure becomes most pronounced in the flat part of the plateau; see Fig. 1(c). There seems to be clear correlations between different layers. At the end of the plateau, cylinders evolve by connecting the dark regions in one of the diagonal directions of the xy plane. The final HEX structure is shown in Fig. 1(d). It is remarkable that this structure is nearly perfect, i.e., free of any structural defects, although a symmetry breaking is clearly required to select the orientation of the cylinders within the lamellar layers.

The structural characteristics of the intermediate structure resemble in many respects the so-called perforated or modulated lamellar phase reported in literature [31-33]. One may tentatively identify the structure in Fig. 1(b) as the modulated lamellae (ML) and that in Fig. 1(c) as the perforated lamellae (PL) although the evolution from ML to PL is a continuous process. It will be shown in Sec. III that the second plateau is due to another saddle point in the free energy surface. The PL structure corresponds to this saddle point, whereas the ML is a transient state between the LAM and PL states. Analyses there show that the perforated lamellar plateau exists only when the final temperature is within a certain "phase boundary" in the HEX part of the phase diagram. This saddle point disappears when the temperature jump is outside of this "phase boundary" as is the case for $\tau_{f}=0.22$. In that case, the cylinder phase emerges directly without going through a distinct PL stage, although a short transient ML state is still involved when the plateau LAM decays.

\section{B. LAM to BCC transition}

The initial state is the same as in the LAM to HEX transition. The temperature parameter is changed from $\tau_{i}=0.35$ to $\tau_{f}=0.216$. The temporal evolution of the global order parameter is shown in Fig. 3. Notice the large initial decrease in the order parameter. This initial fast decrease is followed by a long valley where the order parameter increases only slightly but overall remains very weak. Gray-level plots 


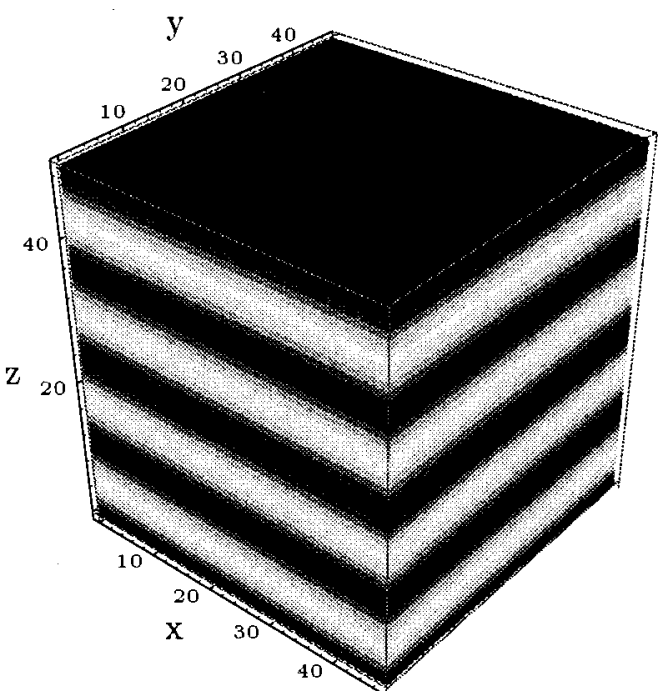

(a) $t=0$ step

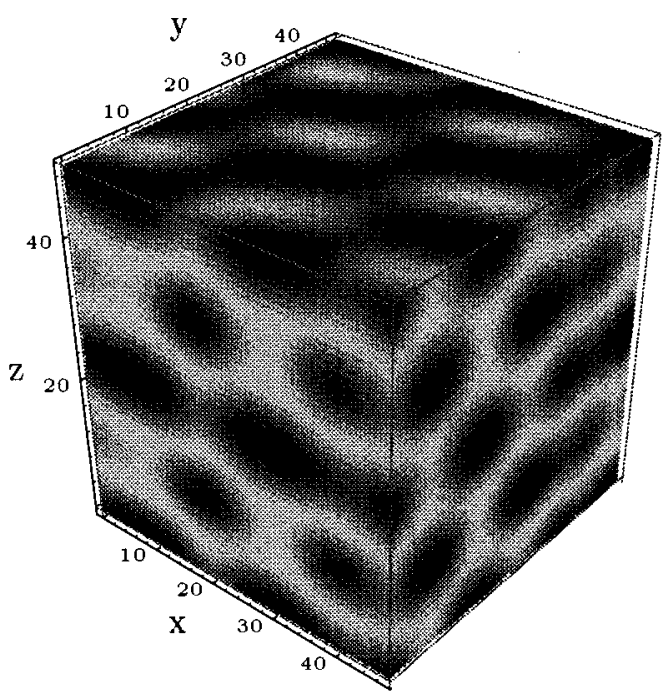

(c) $\mathbf{t}=4000$

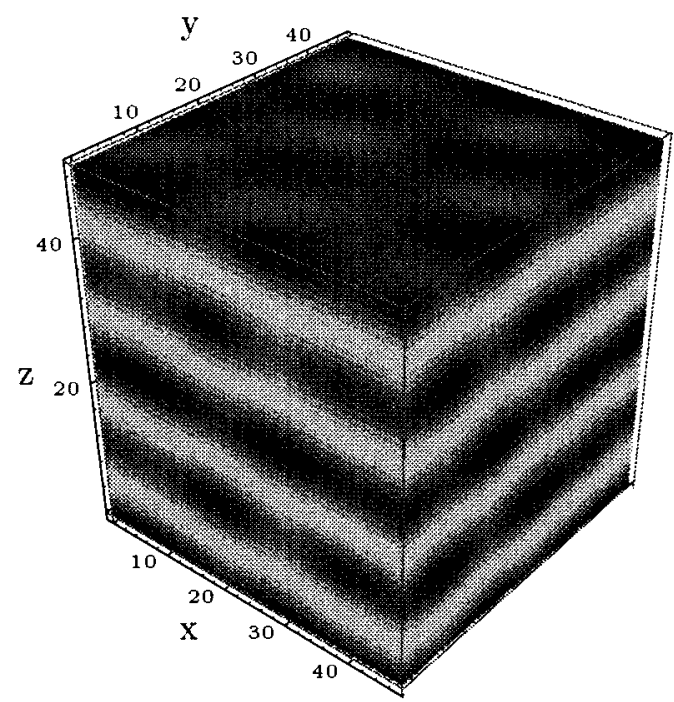

(b) $t=3300$

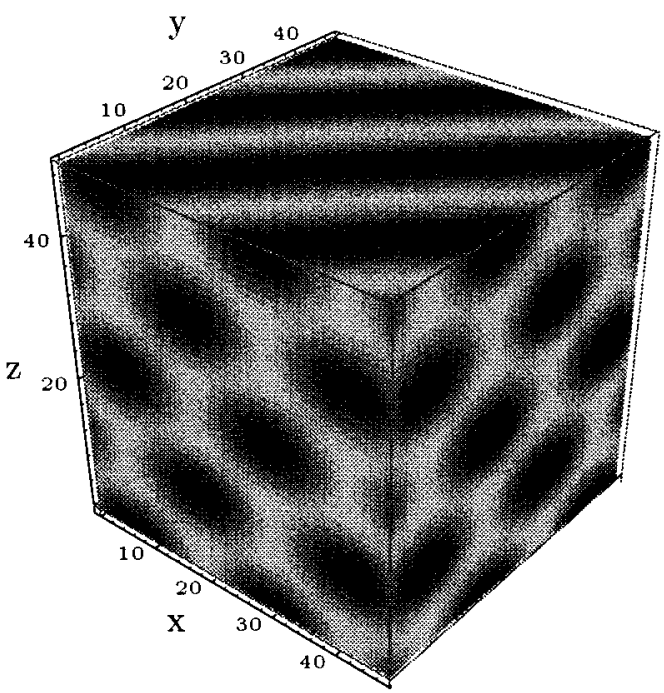

(d) $\mathrm{t}=8000$

FIG. 1. Microstructural evolution after a $T$ jump from LAM to HEX at $f=0.45\left(\tau_{i}=0.35, \tau_{f}=0.25\right)$; data taken at various stages of the simulation.

[with the same contrast level as in Fig. 1(a)] of the spatial distribution of the order parameter shows no specific structure within the lamellar layers at the bottom of the curve, corresponding to $t=5000$ [34]. However, upon enhancing the contrast in the images, some weak traces of the BCC wave superposed on the lamellar structure can be detected. The BCC waves appear at an accelerated pace at about $t=15000$ and the final BCC structure is completed within a short period of time thereafter. It will again be shown that the long valley is due to the existence of saddle regions in the free energy surface. At $t=20000$, the system reaches a stable BCC microstructure, see Fig. 4. Note again the absence of defects in the structure.

\section{LAM to DIS transition}

Using the same initial condition as in Sec. II A and Sec. II B [Fig. 1(a)], we studied two temperature jumps, from

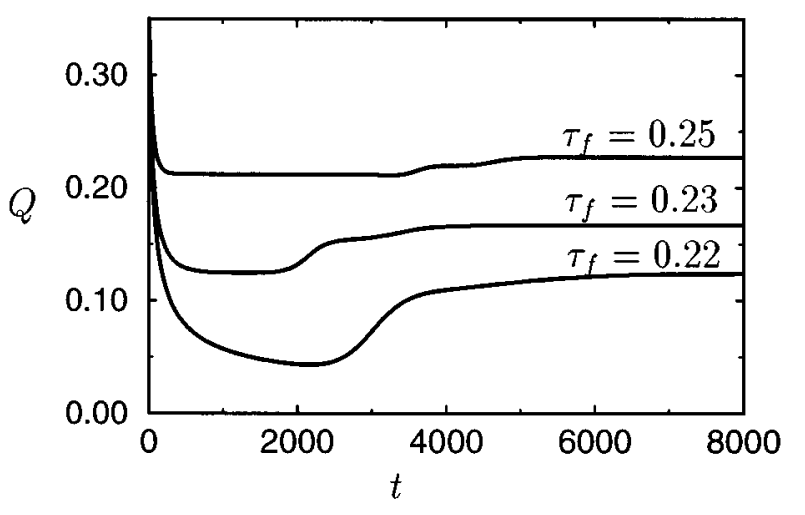

FIG. 2. Temporal evolution of the global order parameter $Q$ during the LAM to HEX transition at $f=0.45$ for $T$ jumps from $\tau_{i}=0.35$ to $\tau_{f}=0.25,0.23$, and 0.22 , respectively. 


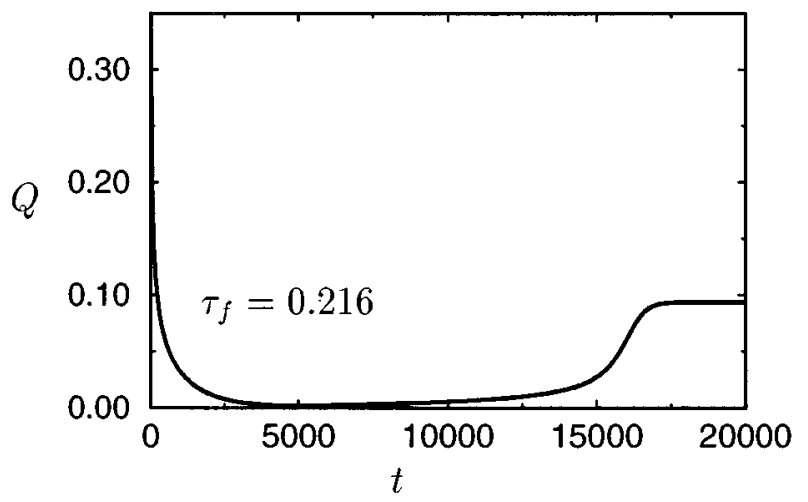

FIG. 3. Temporal evolution of the global order parameter $Q$ during the LAM to BCC transition at $f=0.45$ for a $T$ jump from $\tau_{i}=0.35$ to $\tau_{f}=0.216$.

$\tau_{i}=0.35$ to $\tau_{f}=0.21$ and $\tau_{f}=0.202$, respectively. Both $\tau_{f}$ s correspond to the disordered (DIS) phase. In both cases, the global order parameter decreases monotonically. However, visualization of the gray-level plots of the order parameter during the melting process reveal that melting proceeds through two different routes for the two cases: while in the case of large temperature jump the lamellae melt directly (i.e., the amplitude of the lamellar wave simply decreases), in the case of the smaller temperature jump, the lamellae melt by first developing modulation within the lamellar layers and melt as this three dimensionally ordered structure. Figure 5 shows the gray-level plots in the $x y$ cross section (i.e., in the lamellar layers). Although the exact symmetry of this intermediate structure is difficult to ascertain owing to the small system size used in the simulation and the weakness of the structure, it is reasonable to believe that this structure is the same as the intermediate, modulated lamellar structure observed during the transition from lamellae to

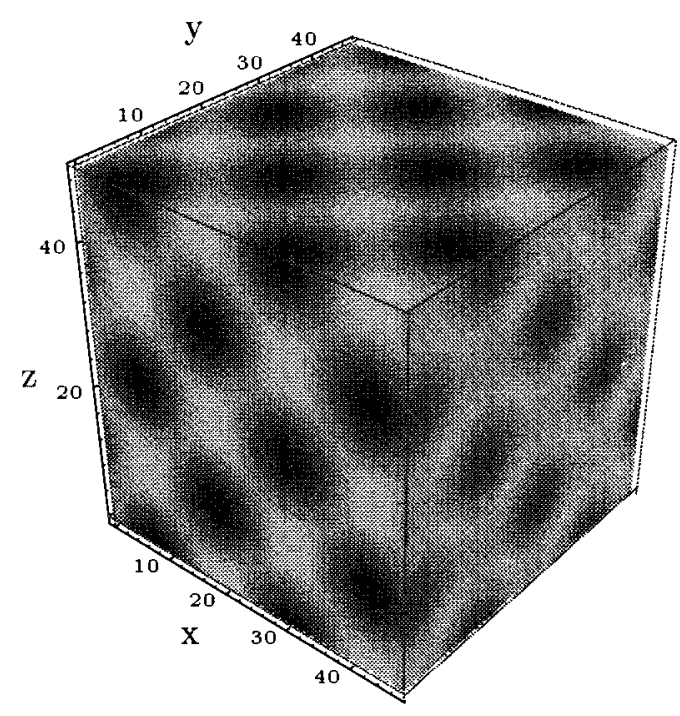

FIG. 4. Final microstructure after a $T$ jump from LAM [same initial state as in Fig. 1(a) $]$ to $\mathrm{BCC}$ at $f=0.45 \quad\left(\tau_{i}=0.35\right.$, $\left.\tau_{f}=0.216\right)$.

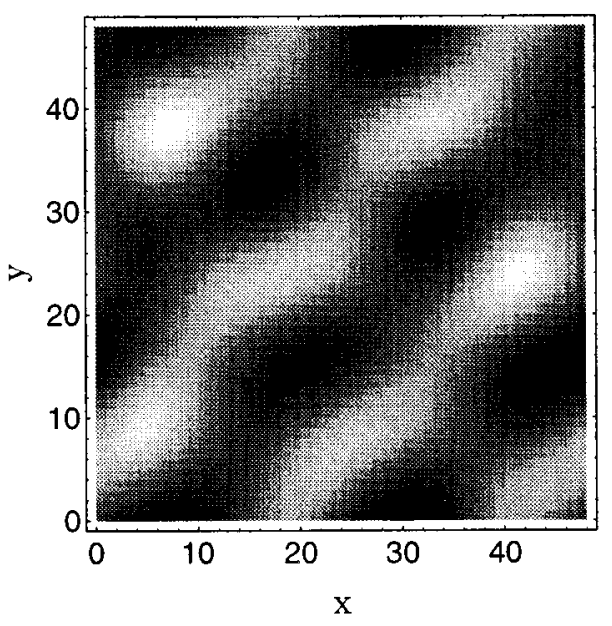

FIG. 5. Cross-section view of the in-layer ( $x y$ plane) structure at $\mathrm{t}=2000$ during the LAM to DIS transition at $f=0.45$ for $\tau_{f}=0.21$.

hexagonal cylinders. Using this assumption, we can show that this transient structure results from a ridge-like landscape in the free energy surface, similar to the transient sphere structure seen during the melting of the hexagonal cylinders [26]; see Secs. III C and III E. On the other hand, the melting for the large temperature jump follows a simple downhill path in a simple parabolic free energy surface.

\section{HEX to BCC transition}

The phenomenology for the HEX to BCC and HEX to DIS transitions has been reported in our previous publication [26]. Here we discuss these transitions both for completeness and to include other details that were omitted in our previous paper. For these two transitions, we choose $f=0.4$ instead of $f=0.45$ used in the previous transitions [35].

An initial equilibrium hexagonal cylinder phase is prepared at $\tau_{i}=0.30$ [see the initial structure in Fig. 7(a)]. Two temperature jumps to $\tau_{f}=0.25$ and 0.246 , respectively, are studied. The evolution of the global order parameter $Q$ is shown for the two cases in Fig. 6.

As with temperature jumps from the LAM phase, a temperature jump from the HEX phase to the BCC phase leads to a rapid initial decrease in the order parameter. This rapid decrease is followed by a plateau which is again due to a saddle point in the free energy. At this saddle point, the system remains a HEX phase. Thus there is a time lag before the BCC structure appears. The sphere structure begins to emerge from the cylinders at the end of the plateau; see Fig. 7(b). The final state is shown in Fig. 7(c). Both temperature jumps exhibit the same qualitative behavior, although the duration of the plateau shortens for the higher temperature (small $\tau$ ). This implies that the saddle point becomes more unstable. Indeed, we have determined that there exists a boundary within the BCC phase, such that the saddle point no longer exists for a temperature jump beyond this boundary. It is interesting to note that, as in the case of LAM to BCC transition, existence of such a saddle point does not 


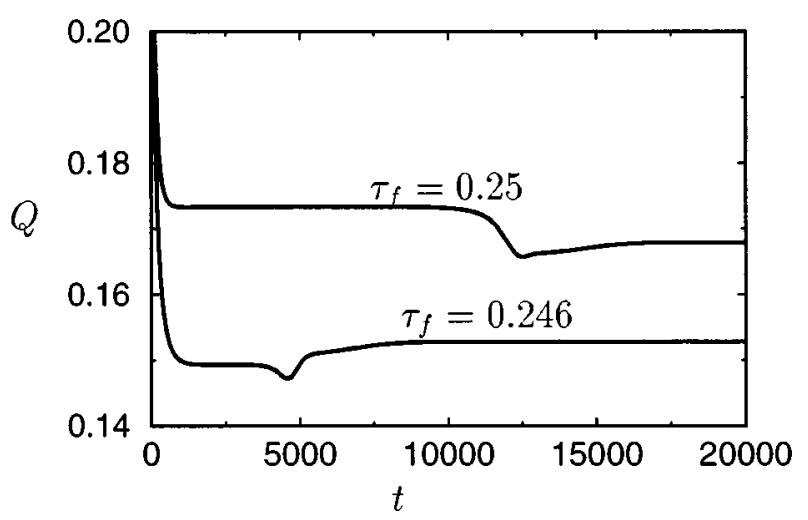

FIG. 6. Temporal evolution of the global order parameter $Q$ during the HEX to BCC transition at $f=0.4\left(\tau_{i}=0.30\right)$.

correspond to a new distinct, intermediate structure, but changes the temporal behavior of the kinetics.

\section{E. HEX to DIS transition}

The same initial state as used in Sec. II D is used as the initial state for the HEX to DIS transition. Two temperature jumps to $\tau_{f}=0.24$ and to $\tau_{f}=0.22$, respectively, are studied. The evolution of the global order parameter is depicted in Fig. 8. For the larger temperature jump $\tau_{f}=0.22$, the order parameter decays in a simple manner, suggesting direct melting of the cylinders. Gray-level plots of the order-parameter at various times show no structural change as the cylinders melt.

In contrast, the behavior of $Q$ in the case of the smaller temperature jump (to $\tau_{f}=0.24$ ) suggests a nontrivial melting process. The slower decay at intermediate times is accompanied by the formation of structures along the cylinders: the cylinders seem to first break into spheres and the melting proceeds via this modulated hexagonal cylinder (MHC) phase. This transient structure is clearest between the two inflection points on the curve, because at long times the structure has all but melted and at short times the structure is dominated by the hexagonal cylindrical structure of the initial state. Figure 9 shows the transient MHC state.

To study the effects of in situ thermal fluctuations, we have performed simulations both with and without these fluctuations and found that the same qualitative behavior is observed. (The results presented in this subsection for the smaller temperature jump have been obtained with thermal fluctuations: the final nonvanishing value of $Q$ in Fig. 8 is due to these fluctuations.) However, the inclusion of thermal fluctuations in the case of the smaller temperature jump is found to enhance the transient modulated cylinder structure. This point will be discussed further in Sec. IV A.

\section{F. BCC to DIS transition}

For this transition, we chose $f=0.45$ and use the final BCC structure obtained in the LAM to BCC transition and the corresponding $\tau_{i}=0.216$; see Fig. 4 . Two temperature jumps are studied with $\tau_{f}=0.21$ and $\tau_{f}=0.202$, respectively. The behavior of the global order parameter shows some slight difference for the two temperature jumps. For the smaller temperature jump ( $\left.\tau_{f}=0.21\right)$, the curve has two inflection points, while for the larger temperature jump $\left(\tau_{f}=0.202\right)$, the decay is rather fast and is nearly exponential. This difference for the larger and smaller temperature jumps in this case is rather similar to the difference in the behavior of $Q$ for the larger and smaller temperature jumps in the LAM to DIS and HEX to DIS transitions. However, in this case, there is no free energy ridge. In Sec. IIIF we will show that there exist two inflection points along the kinetic path on the free energy surface. Although this does not lead to any new intermediate structures (the system melts as a perfect BCC structure), it changes the temporal behavior of the melting process.

\section{MODE ANALYSES USING A SINGLE-WAVE-NUMBER APPROXIMATION}

To understand the observations of the numerical results and to gain insight into the transition mechanisms, in this section we perform a simplified single-wave-number, amplitude equation analysis [36]. This analysis is motivated by our observation that to a good approximation the kinetics of the various transitions can be described by the development and disappearance of various density waves. In fact nearly all the transient structures studied in this paper can be represented by an appropriate superposition of the LAM, HEX, and BCC waves, and the time evolution of the system corresponds to a time evolution of these waves with generally changing proportions. This conclusion is reached both by direct visual inspection of the structures and by Fourier analyses of several transient microstructures into the dominant modes. The single-wave-number assumption is justified because density modulations in the weak segregation limit is nearly sinusoidal.

We seek a minimal set of wave vectors which can represent the LAM, HEX, BCC, as well as intermediate states observed in the preceding section. To this end, we choose the set of wave vectors corresponding to the first smallest wavevectors of a bcc lattice whose magnitude we take to be that of the optimal wave vector $k^{*}=(c / b)^{1 / 4}$ [37] determined from the quadratic term of the free energy. The order parameter is then represented by $\psi=\sum_{n=1}^{6} A_{n} \cos \left(\overrightarrow{k_{n}} \cdot \vec{r}\right)$, where $\left\{\overrightarrow{k_{n}}\right\}$ where the $A_{n} \mathrm{~s}$ (generally not equal to each other) are the amplitudes associated with these respective wave vectors. We set our coordinate system such that

$$
\begin{gathered}
\overrightarrow{k_{1}}=k^{*}(0,0,1), \quad \overrightarrow{k_{4}}=\frac{k^{*}}{3}(-\sqrt{6}, \sqrt{3}, 0), \\
\overrightarrow{k_{2}}=\frac{k^{*}}{2}(0, \sqrt{3}, 1), \quad \overrightarrow{k_{5}}=\frac{k^{*}}{2 \sqrt{6}}(-4,-\sqrt{2}, \sqrt{6}),
\end{gathered}
$$

$$
\overrightarrow{k_{3}}=\frac{k^{*}}{2 \sqrt{6}}(4, \sqrt{2}, \sqrt{6}), \quad \overrightarrow{k_{6}}=\frac{k^{*}}{2}(0,-\sqrt{3}, 1)
$$

We choose the lamellar normal to be in the direction $\overrightarrow{k_{1}}$. 

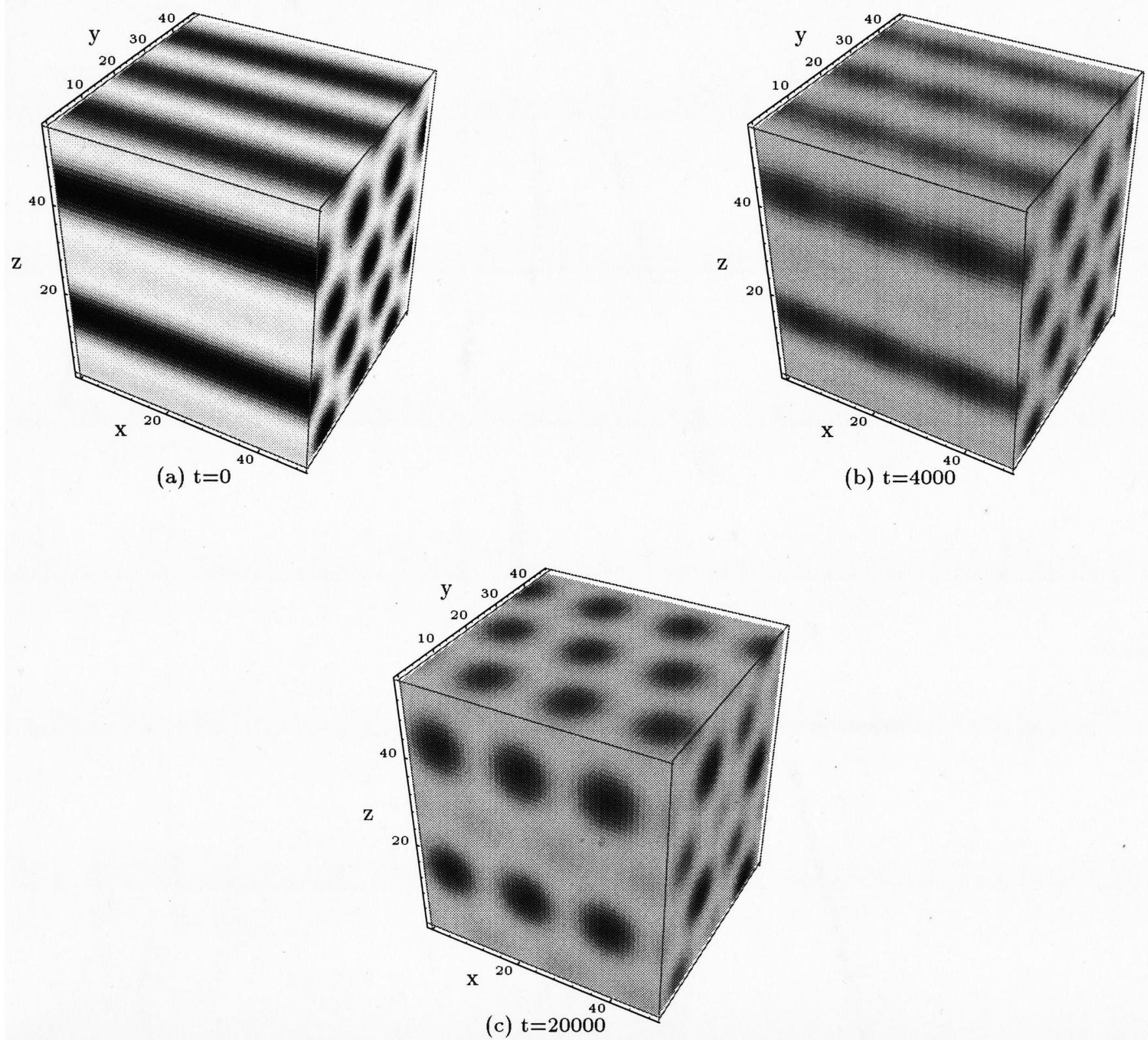

FIG. 7. Microstructural evolution during the HEX to BCC transition at $f=0.4$ with $\tau_{i}=0.30$, and $\tau_{f}=0.246$.

Thus, in this single-wave-number approximation, the LAM structure is represented by $A_{1} \neq 0$ and $A_{n}=0(n=2-6)$. The HEX structure can be obtained by choosing any three coplaner waves that include $\overrightarrow{k_{1}}$ with equal amplitude, while the BCC structure requires all six $A_{n}$ s to be equal in magnitude [38] and nonvanishing. In this construction, the "epitaxial", relationship between the initial and final structures [39], is automatically satisfied. For example, the HEX phase grown from the LAM phase will have the cylinder axes lying within the initial lamellar layers, and the spheres of the BCC phase emerging from the HEX phase will reside on the initial cylinder axes.

In terms of the amplitudes $A_{n} \mathrm{~s}$, the free energy can be written as

$$
\begin{aligned}
F= & {\left[-\tau+a(1-2 f)^{2}+2 \sqrt{b c}\right] \sum_{n=1}^{6} A_{n}^{2}+4 v(1-2 f)\left(A_{1} A_{2} A_{6}\right.} \\
& \left.+A_{1} A_{3} A_{5}+A_{2} A_{3} A_{4}+A_{4} A_{5} A_{6}\right)+12 u\left(A_{1} A_{2} A_{4} A_{5}\right. \\
& \left.+A_{2} A_{3} A_{5} A_{6}+A_{1} A_{3} A_{4} A_{6}\right)+6 u \sum_{n=1}^{6} \sum_{m>n}^{6} A_{n}^{2} A_{m}^{2} \\
& +1.5 u \sum_{n=1}^{6} A_{n}^{4} .
\end{aligned}
$$

Correspondingly, the dynamic equation Eq. (1) becomes a set of equations for the various amplitudes [36] 


$$
\frac{\partial A_{n}}{\partial t}=-M k^{* 2} \frac{\partial F}{\partial A_{n}} ; \quad(n=1-6) .
$$

Note that in the single-wave-number approximation, the Laplacian operator in Eq. (1) is transformed to $-k^{* 2}$ [37] , a constant factor. Thus Eq. (6) simply describes the steepest descent of the order parameters on the free energy surface given by Eq. (5). This allows us to investigate the kinetics of the transitions by studying the landscape of the different free energy surfaces corresponding to the different transitions.

Further symmetry considerations require that $A_{2}=A_{6}$ and $A_{3}=A_{5}$. Thus we group the six waves into four subsets: (i) $\overrightarrow{k_{1}}$, with amplitude $A_{1}=A$; (ii) $\overrightarrow{k_{2}}$ and $\overrightarrow{k_{6}}$, with amplitudes $A_{2}=A_{6}=B$; (iii) $\overrightarrow{k_{3}}$ and $\overrightarrow{k_{5}}$, with amplitudes $A_{3}=A_{5}=C$; and (iv) $\overrightarrow{k_{4}}$, with amplitude $A_{4}=D$. The various transient structures can be obtained by the appropriate superposition of these four subsets of waves.

The perforated-modulated lamellar (PL-ML) structure shown in Sec. II A deserves some further remarks. Within the bcc-based, single-wave-number approximation, periodic density modulations in the lamellar layers can be represented by a structure with $-A>B=C>-D>0$. Such a structure does not have perfect in-layer sixfold symmetry which is believed to be associated the PL-ML structure [31]. We have also used a hcp(hexagonal-close-packing) based wave vector set to describe the LAM to HEX transition and found the same qualitative behavior as we did using the bcc-based lattice in all cases where a transient perforated lamellar structure is involved. Thus, we will adhere to the bcc-based representation in most part of this paper as it enables to treat all the structures considered in a unified framework. In fact, there is reason to believe that experimentally observed PL-ML structures may be either hcp or bcc based [40].

\section{A. The LAM to HEX transition: pseudostable LAM and PL}

In this phase transition, all the four groups of waves $A$, $B, C$, and $D$ are involved, where as mentioned above, $A$ is the amplitude of the initial lamellar wave. Either $A=-B$ or $A=-C$ (where the minus sign ensures the proper phase relationship) can form a HEX structure given that the other parameters are zero, and when $A, B, C$, and $D$ are all non-

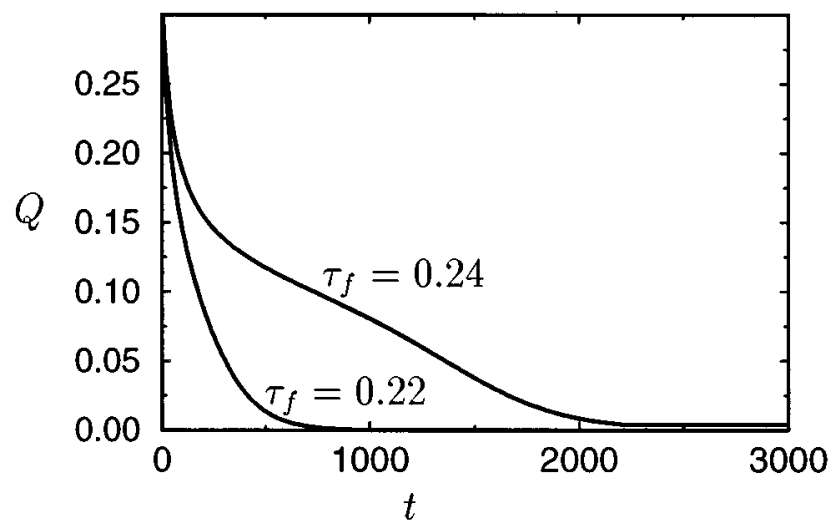

FIG. 8. Evolution of the global order parameter $Q$ for the $T$ jump from the HEX to DIS phase at $f=0.4$ with $\tau_{i}=0.30$.

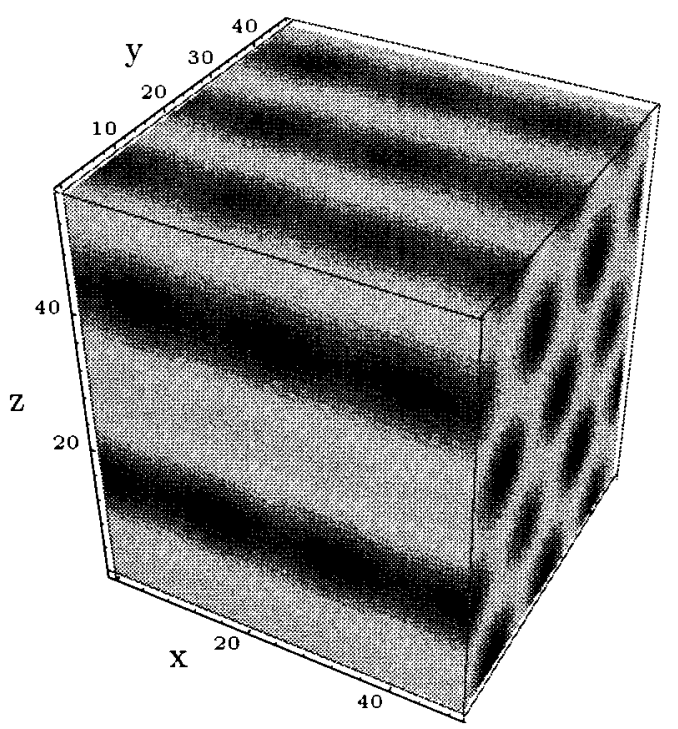

FIG. 9. Intermediate state during the HEX to DIS transition at $f=0.4\left(\tau_{i}=0.30, \tau_{f}=0.24\right)$, taken at $\mathrm{t}=1000$.

zero, a three-dimensional periodic structure is obtained. In particular, $-A>B=C>-D>0$ represent a structure with density modulations within the lamellar layer; we will identify this structure as the perforated-modulated lamellar (PLML) structure.

The kinetic pathway from the LAM phase to the HEX phase after changing $\tau$ from 0.35 to 0.25 is shown by the time evolution of the four waves, see Fig. 10. Initially $A=-0.3$ and all the other three amplitudes are set to zero (some small perturbations are included as initial driving forces for these waves to emerge.). The final state with $A=-B$ and $C=D=0$ clearly corresponds to the HEX structure. Note, however, the existence of two plateau regions. In the first plateau region, $A$ is nonzero, although it has decreased significantly from its initial value. But the other three wave amplitudes are still nearly zero. Therefore the structure corresponding to this plateau is still lamellar. The length of this plateau depends on the initial perturbation in the other

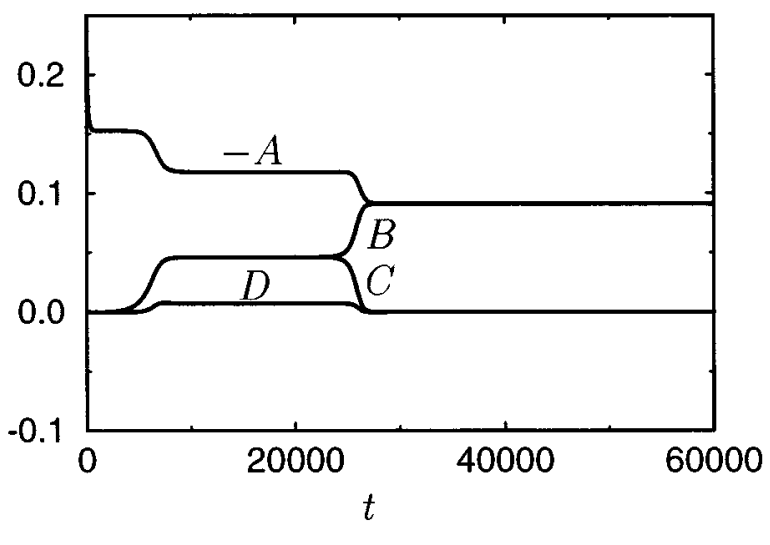

FIG. 10. Evolution of the wave amplitudes during the LAM to HEX transition at $f=0.45\left(\tau_{i}=0.35, \tau_{f}=0.25\right)$. Note the appearance of two plateaus beside the final HEX state. 


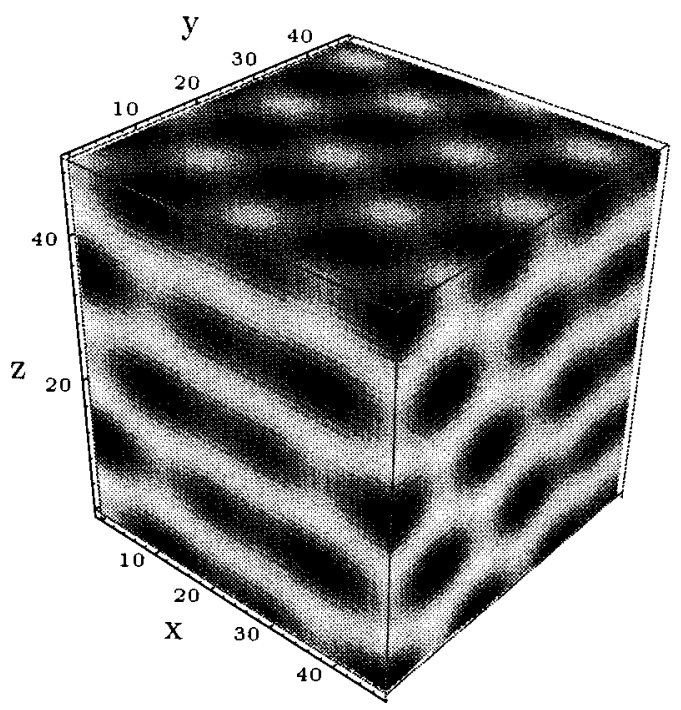

FIG. 11. Microstructure generated using the six wave amplitudes with values corresponding to the second plateau in Fig. 10.

three waves and on the temperature. At the end of the first plateau, $A$ has another quick drop, and at the same time the other three waves take off. Then the system reaches the second plateau. On this plateau, $D$ remains quite small, but $B$ and $C$ have increased considerably while maintaining $B=C$. The structure corresponding to this second plateau is shown in Fig. 11. It is rather reminiscent of the perforated lamellar structure Fig. 1(c) observed in the numerical simulation. The results in Sec. II A, including the behavior of the global order parameter and the microstructural features during the transition, are all consistent with the foregoing analysis.

The plateaus in Fig. 10 suggest that the system passes near saddle points on the free energy surface. Using the free energy Eq. (5), we can locate these saddle points and the region of the phase diagram in which they exist. We find that the first saddle point evolves from the local free energy minimum corresponding to the LAM phase, i.e., the metastability limit of the LAM phase is reached by turning the local minimum into a saddle point. This scenario is shown in the reduced parameter space of $A$ (the lamellar wave) and $B$ (which, together with $A$, consititutes the HEX wave) in Figs. 12(a) and 12(b) with $C=D=0$. The value of $\tau$ where this happens marks the spinodal of the lamellar phase. We shall term states corresponding to saddle points in the free energy surface pseudostable states [30]. The range of $\tau$ in which this saddle point exits is shown schematically in Fig. 15. Because of the positive curvature along the $A$ axis in Fig. 12(b), the decay of the initial LAM structure (with $A$ more negative than its saddle point value) in this temperature range, will follow a path that drives the system to the saddle point. This saddle point is responsible for the near stagnation (plateau) in both Fig. 2 and Fig. 10. As the temperature increases $(\tau$ decreases), the negative curvature in the $B$ direction increases and the positive curvature in the $A$ direction decreases, thus the time spent by the system near the saddle point gets shorter. This is indeed the case; see Fig. 2.

A similar saddle point is responsible for the appearance of the second plateau in Fig. 10. At this saddle point, however, all the three amplitudes $B, C$, and $D$ are nonzero, but $B=C$. Therefore the structure has three dimensional rather than one-dimensional order. The saddle point is unstable with respect to bifurcation from $B=C$. This is shown in Fig. 12(c) where we set $A=-0.117808$ and $D=0.007061$ at their respective saddle point values. The system eventually leaves this saddle point following the unstable direction which leads to either $A=-B, C=D=0$, or $A=-C$, $B=D=0$, both respresenting a perfect HEX structure with the cylinder axes lying within the original lamellar layers. The range of $\tau$ where this saddle point exists is shown schematically in Fig. 15, which lies within the HEX part of the equilibrium phase diagram.

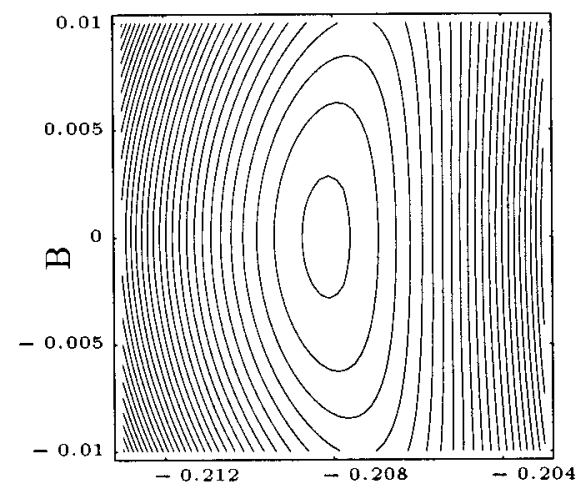

A

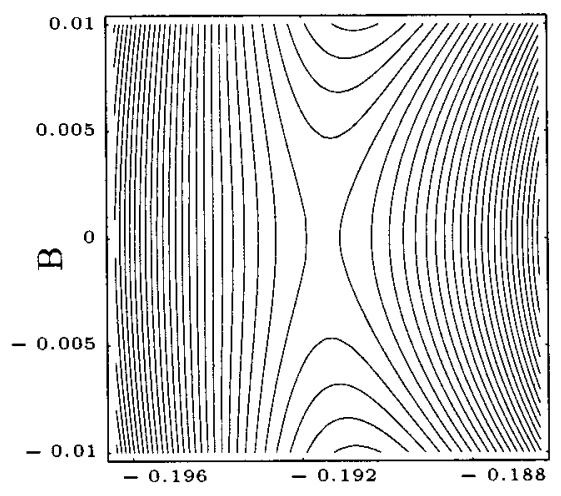

A

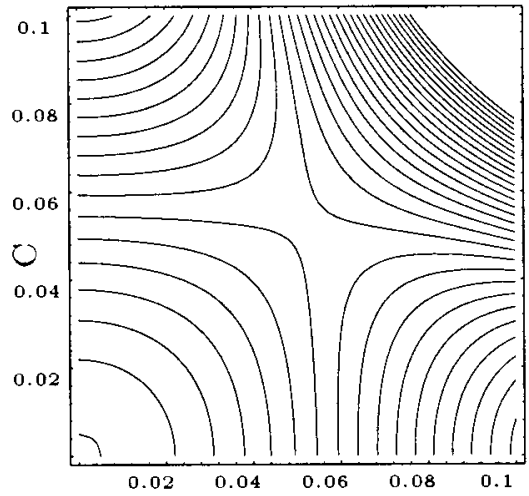

B

FIG. 12. Free energy contour plot in reduced order parameter space illustrating (a) the metastable LAM ( $\left.\tau_{f}=0.28\right)$, (b) the pseudostable $\operatorname{LAM}\left(\tau_{f}=0.27\right)$, and (c) the pseudostable PL $\left(\tau_{f}=0.25\right)$ states. In (a) and (b) $C$ and $D$ are both set to zero whereas in (c) $A$ and $D$ are set to the saddle point values $(A=-0.11781, D=0.00706) . f=0.45$ for all three figures. 
Although the pseudostable LAM structure itself has no interesting structural features, the decay from this structure is nontrivial. It turns out the most unstable direction is the direction of increasing $B$ and $C$ with $B=C$. This implies that when the system leaves the pseudostable LAM, it does so by going through a transient ML state. If a pseudostable PL state exists, the ML will evolve to the PL state which eventually decays to the HEX. If the temperature is outside of the pseudostable PL region, the ML will evolve continuously to the HEX structure without a distinct intermediate stage. These conclusions are indeed consistent with the results in Sec. II A.

\section{B. LAM to BCC transition: pseudostable LAM states}

As in the LAM to HEX transition, all the four waves $A$, $B, C$, and $D$ are required to describe this transition. The kinetic pathway displays two different behaviors depending on the extent of the temperature jump. For the smaller temperature jump from $\tau_{i}=0.35$ to $\tau_{f}=0.216$, the kinetic pathway shows a plateau similar to the first plateau in the LAM to HEX transition. At this plateau the structure is again lamellar with a reduced wave amplitude. The other three waves emerge almost equally at the end of this plateau while $A$ continues to decrease, reaching the final value $-A=B=C=-D=0.02824$ corresponding to the equilibrium $\mathrm{BCC}$ structure at this temperature.

For the larger temperature jump from $\tau_{i}=0.35$ to $\tau_{f}=0.212$, no plateau is seen in the pathway: there is a steady decrease of $A$ from its initial value and a steady increase of $B, C$, and $D$, reaching the steady-state value of 0.02488 . Incidently, we find that relaxation to the final equilibrium BCC structure is slower for the case of the larger $T$ jump; this is to be expected because the final temperature is closer to the (mean-field) spinodal (of the DIS phase).

The LAM saddle point for the smaller $T$ jump in the LAM to $\mathrm{BCC}$ transition has the same origin as the LAM saddle point in the LAM to HEX transition. Beyond the spinodal of the LAM phase, the LAM saddle point (stable with respect to variation in the lamellar wave amplitude $A$ but unstable with respect to perturbations in at least one of the other amplitudes) persists over a rather wide region in the phase diagram; this region has overlap with the lower temperature part of the equilibrium BCC phase. Thus if the $T$ jump is into this region, transition from the LAM to the BCC phase will proceed by first going through the LAM saddle point with the consequence of a time lag in the growth of the BCC structure. Beyond this region, transition will be a direct one without a time lag. The scenario described in this section is fully consistent with the simulation results of Sec. II B.

\section{LAM to DIS transition: transient ML state}

We again start with the equilibrium LAM structure at $\tau_{i}=0.35$ with $A=-0.3$. Two $T$ jumps to $\tau_{f}=0.21$ and $\tau_{f}=0.202$ are studied by numerically solving the four coupled amplitude equations with small perturbations in $B$, $C$, and $D$ of the order of $0.3 \%$ of the initial value of $A$. The evolution of $A, B, C$, and $D$ at $\tau_{f}=0.21$ is shown in Fig. 13 . All these four amplitudes eventually decay to zero as the equilibrium phase at this $\tau$ is the DIS phase. However, note the transient increase in $B$ and $C$. At the maximum of $B$ and

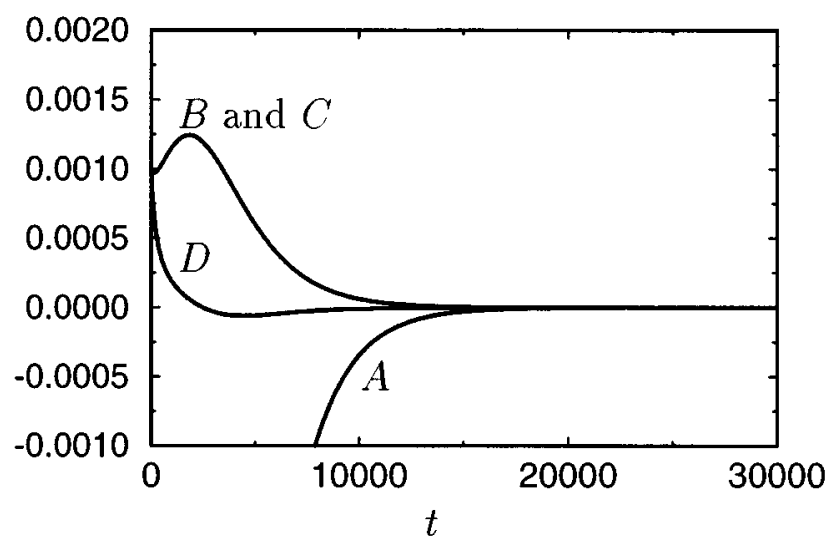

FIG. 13. Temporal evolution of the different modes during the transition from LAM to DIS at $f=0.45\left(\tau_{i}=0.35, \tau_{f}=0.21\right)$.

$C$, the structural feature is similar to that obtained during the transition from the LAM to HEX, although the density modulation within the layer (as measured, for example, by $|B / A|$ or $|C / A|)$ is much weaker here. For this reason we term this transient structure also ML. Although the structural features between this ML and the ML observed in the LAM to HEX transition are the same, the mechanisms which give rise to this structure in the two cases are different. Recall that in the case of the LAM to HEX transition, the ML is due to a saddle point on the kinetic path: it is the most unstable mode for the pseudostable LAM to decay, and if a pseudostable PL state exists, it will further evolve into such a state. Here, however, no such saddle points exist. Rather, the transient growth of the amplitude $B$ or $C$ is due to deflection from the path along the axis $B=C=D=0$ caused by a ridgelike feature in the free energy landscape. This is shown by a contour plot of the free energy in the reduced parameter space $A, B$, see Fig. 14. We have found analytically that there exists a small finite window $\tau_{l}<\tau<\tau_{u}$ in the phase

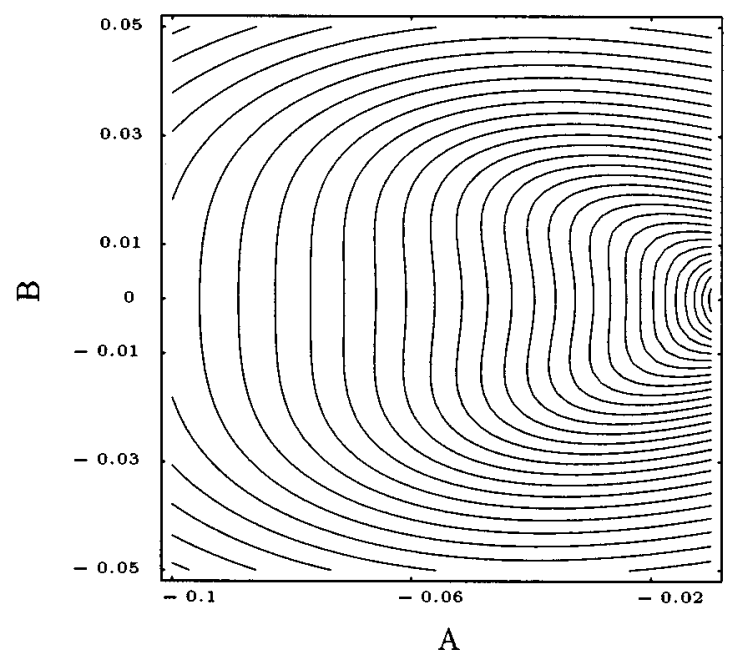

FIG. 14. Free energy contour plot in the reduced order parameter space showing the ridgelike feature during the LAM to DIS transition at $f=0.45\left(\tau_{f}=0.21\right) . C$ and $D$ are both set to zero. 
diagram for this transient state to exist. The upper boundary $\tau_{u}$ coincides with the BCC-DIS phase boundary. For $f=0.45, \tau_{l}=0.2075$. Therefore, when $\tau_{f}=0.202<\tau_{l}$, all four amplitudes decrease monotonically, and melting of the LAM will proceed directly without involving a transient ML state. This compares well with the simulation results.

\section{HEX to BCC transition: pseudostable HEX state}

Assuming that this transition involves only the initial HEX waves and another set of waves which are part of the BCC structure, the four groups of waves can be further reduced to two, denoted by $A(=B)$ and $C(=D)$, respectively, where $A$ consititutes the HEX density wave, with $A=A_{1}=A_{2}=A_{6}$ and $C=A_{3}=A_{4}=A_{5}$.

The temporal evolution of $A$ and $C$ for a $T$ jump from $\tau_{i}=0.30$ to $\tau_{f}=0.25$ at $f=0.4$ was reported in our earlier publication, and will not be shown here. It suffices to note that there is a long plateau after the rapid decrease in $A$ from its initial value. Within the duration of this plateau, $C$ remains close to zero. Thus there is a time lag for the BCC structure to appear, and when it does, the structure becomes that of a modulated hexagonal cylinder (MHC) with a continuous growth of the BCC component, reaching the final equilibrium state with $A=-C$. The plateau arises from a saddle point which lies on the $C=0$ axis. Thus we have a pseudostable HEX structure in the HEX to BCC transition, similar in its origin to the pseudostable LAM state. Once again, there is a region in the phase diagram for the pseudostability. The lower temperature (larger $\tau$ ) side of the boundary is the spinodal of the HEX phase, i.e., when the HEX reaches its metastability limit, it does so by turning the local minimum of free energy into a saddle point. The situation is similar to how the LAM phase loses its metastability, see Sec. III A. The high- $T$ (lower $\tau$ ) boundary lies within the BCC part of the phase diagram: for $f=0.4, \tau_{c}=0.248$. Therefore a $T$ jump to a $\tau_{f}$ value beyond this boundary, i.e., $\tau_{f}<\tau_{l}$, but still in the BCC part of the phase diagram leads to a direct transition from the HEX to the BCC. This explains the observations in Sec. IID.

\section{E. HEX to DIS transition: transient MHC state}

In this case, the kinetics is again described by the two order parameters $A$ and $C$, as defined in the last subsection. For a large $T$ jump ( $\tau_{i}=0.30$ to $\left.\tau_{f}=0.22\right), A$ decreases monotonically to zero; also any initial perturbation from $C=0$ is rapidly damped. This corresponds to a direct melting of the HEX phase.

For a smaller $T$ jump ( $\tau_{i}=0.30$ to $\tau_{f}=0.24$ ), while $A$ still decreases monotonically, an initial perturbation from $C=0$ first grows before it decays again to zero. Thus there is a transient growth of the BCC component of the density wave. This wave, superposed on the dominant HEX wave, results in a modulated hexagonal cylinder (MHC) state. These results are in full agreement with the simulation results.

By studying the free energy landscape in the order parameters $A$ and $C$, we find that there exists a window in the phase diagram in which the landscape exhibits a ridgelike feature. The transient appearance of the BCC feature during the melting of the HEX is thus caused by the deflection of the kinetic path from the $C=0$ ridge.
The degree of the deflection from the ridge, and hence the strength of the modulation and the length of its duration, is closedly related to the magnitude of the fluctuations. In the absence of any fluctuations (including initial noise), a path that starts with $C=0$ will keep $C=0$ even though the $C=0$ axis is a ridge. Deviation from $C=0$ is caused by perturbations (either given initially or included in situ). The same considerations apply to other transient phenomena caused by ridgelike free energy landscapes. This issue will be discussed more fully in Sec. IV.

\section{F. BCC to DIS transition}

The transition from BCC to DIS is relatively featureless. Here, we do not find saddle or ridgelike features in the free energy landscape. The numerical simulation above does not show any distinctive intermediate structure. However, upon closer investigation of the free energy, we find two inflection points on the otherwise downhill path in a temperature window beyond the spinodal limit of the BCC phase. The high$T$ (low $\tau$ ) boundary of this window corresponds to $\tau_{l}=0.2097$ at $f=0.45$. For $\tau_{f}<\tau_{l}$ the order parameter will decay as a simple exponential, whereas for $\tau_{f}>\tau_{c}$ the melting will be somewhat slowed down between the two inflection points. We believe this is the reason for the nonsimple decay of the order parameter seen at the larger $\tau_{f}$ in Sec. IIF.

\section{G. Generalized phase diagram}

The different scenarios described in the previous sections can be summarized in a generalized phase diagram. This phase diagram is obtained by a detailed study of the free energy landscape in the four order parameters $A, B, C$, and $D$, because under the assumption that the kinetics of the various order-order and order-disorder transitions can be described by a few dominant modes with a single wave number, the kinetics reduces to a steepest descent path on the free energy surface. We have calculated analytically all the meanfield "phase boundaries" for the transitions studied in this paper in terms of $\tau$ and $f$. However, there are too many curves in this phase diagram to leave any space for labeling the different scenarios. Therefore, we choose to show only the different behaviors at a single $f=0.45$. The qualitative behavior is the same for other values of $f$. The diagram is not drawn to scale; only the correct sequence of these phase boundaries in $\tau$ is maintained.

The phase diagram is shown in Fig. 15. The three thick solid lines represent the equilibrium phase boundaries between the LAM and HEX, HEX and BCC, and BCC and DIS, respectively. These equilibrium phase boundaries are of course independent of the initial state of the system. However, all other boundaries describe the different scenarios during the transition from one phase to another, and hence one must specify the initial state of the system. From left to right, the three columns describe the $T$-jump transition kinetics starting from the LAM, HEX, and BCC phases, respectively.

We now illustrate the richness of the phase diagram by following a $T$-jump sequence from the equilibrium LAM phase. Seven different kinetic scenarios can be identified, depending on the final temperature $\left(\tau_{f}\right)$. If $\tau_{f}$ lies in region $I_{L}$ (the subscript $L$ refers to the initial LAM phase), the 


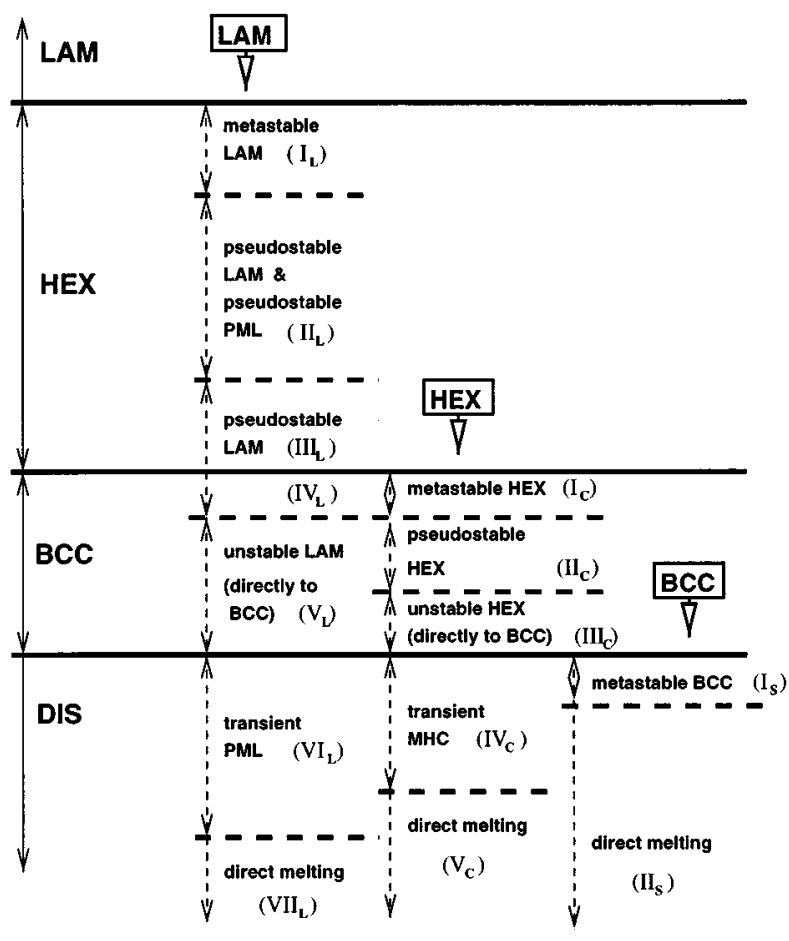

FIG. 15. Generalized phase diagram: the different regions indicate different transition scenarios and the intermediate states involved, starting (from left to right) from LAM, HEX, and BCC phases, respectively.

lamellar phase is metastable. Transition to the HEX phase can only occur via nucleation of the HEX within the LAM phase. The dotted line indicates the spinodal of the LAM phase. In region $I I_{L}$, the LAM phase will first become pseudostable, then passes through another pseudostable state (which we identify as the PL), and finally reaches the HEX state. In region $I I I_{L}$, the LAM state will again first become pseudostable. However, after some time lag spent at this pseudostable state, the HEX phase will emerge gradually (via a short transient ML) but directly from the LAM structure without another clear intermediate stage. In Region $I V_{L}$, the LAM will spend some time at the pseudostable LAM state, and then transform continually to the BCC structure. This transition will again necessarily involve a short transient ML structure, but no distinct stages can be identified. It is interesting, and not coincidental, that the lower boundary of the pseudostable LAM is identical to the spinodal limit of the HEX phase. In Region $V_{L}$, the transition from LAM to BCC will be a direct one. Again, the transition will involve the ML state, but the process is a continuous one with no distinguishable stages. If the final $\tau_{f}$ lies in Region $I V_{L}$, the LAM phase will melt by going through a transient ML state, caused by a ridgelike feature in the free energy landscape which deflects melting of the LAM from following a direct path. Finally, in Region $V I I_{L}$, the LAM phase will melt directly without involving any other structures.

The different kinetic scenarios for transitions starting from the HEX or BCC phases can be similarly identified from reading the generalized phase diagram.

Whereas equilibrium states correspond only to the global free energy minimum, the kinetics of the various transitions involve states that are caused by other features of the free energy surface. Besides the thermodynamically stable structures, there are metastable states corresponding to local free energy minima, pseudostable states corresponding to saddle points, and transient states either arising from deflection of a more direct path or serving to connect to distinct states. Finally unstable states in our study refer to those which possess a nonzero thermodynamic driving force and decays uniformly and monotonically. Clearly, inclusion of these states greatly broadens the phase diagram.

\section{DISCUSSION}

In this paper, we have used a TDGL approach to investigate the kinetics of several order-order and order-disorder transitions after a $T$ jump in weakly segregated block copolymers. Both direct numerical simulation of the TDGL equations and a simplified mode-analysis reveal that the kinetics is far from trivial even in the absence of any free energy barriers. In particular, we propose that the kinetic pathways often involve saddle and ridgelike features in the free energy surface, which can lead to interesting intermediate states and nonsimple temporal evolution of the system. A multitude of kinetic scenarios have been identified. In this section, we discuss the experimental relevance of our findings. Since the presentation of our results in this paper has been in the framework of a mean-field theory, we start with a brief discussion of the effects of fluctuations.

\section{A. Effects of fluctuations}

It is well known that thermal fluctuations have rather profound effects on the microphase transitions near the orderdisorder transition temperature for systems described by free energies of the type Eq. (3). The phenomenology of the fluctuation effects was first worked out by Brazovskii [41]. Fredrickson and Helfand [42] applied the Brazovskii theory to diblock copolymers, where it was shown explicitly that the molecular weight of the diblock plays the role of the Ginzburg parameter: the effects of fluctuations decrease with increasing molecular weight. The main effects of thermal fluctuations are (1) the mean-field second order transition for a symmetric diblock is renormalized to a weakly first order one; and (2) near the symmetric composition, the LAM phase and the HEX phase can be directly accessible from the DIS phase. One would expect these results to have certain bearing on the kinetics. However, as we mentioned earlier, we have conducted studies which include thermal fluctuations in the form of Eq. (2), and did not find the kinetics to be qualitatively different from simulations which only include an initial randomness. It is possible, that the two $f$ 's used in our study $f=0.4$ and $f=0.45$ are already asymmetric enough for the fluctuation effects to become unimportant [43]. More systematic studies are needed to further address this issue. A simulation of the full equilibrium behavior of free energy Eq. (3) by the Monte Carlo method or the TDGL approach used here would seem a welcome effort.

Provided that the qualititative features identified in the mean-field framework are correct, fluctuations (either thermal fluctuations or other externally imposed perturbations) have some systematic quantitative effects on the pseudostable and transient states. Since the pseudostable states correspond to saddle points on the free energy surface, fluc- 
tuations will lead to a faster decay from the saddle point and hence the lifetime of these pseudostable structures become shorter with the inclusion of fluctuations. On the other hand, transient structures that are caused by ridgelike landscapes of the free energy will be enhanced since fluctuations help the sideway pushes which are responsible for the appearance of these transient structures in the first place. The opposite effects of fluctuations on the pseudostable states and transient states caused by ridgelike free energy landscapes could conceivably be exploited to experimentally distinguish between these two types of intermediate states.

\section{B. Comparison with experiments: The nature and stability of the PL-ML structure}

The number of experiments that directly probe the kinetics of microphase transitions described in this paper is limited at present. Hashimoto and co-workers studied the transition from the LAM phase to the DIS phase after a $T$ jump using time-resolved x-ray scattering [44]. However their study was focused on the diffusion of the chains across the order-disorder boundary and not on the microstructural changes. More recent experiments by Singh et al. [45] studied the ordering kinetics of block copolymers after a temperature quench, with a focus on nucleation and growth.

Of more direct relevance to our study is a recent work by Sakurai et al. [46]. These authors studied the HEX to LAM transition in poly(styrene-b-butadiene-b-styrene) triblock copolymers. They used solvent casting to produce a nonequilibrium HEX cylinder phase which was transformed into the LAM phase upon annealing. Based on small-angle X-ray scattering (SAXS) and transmission electron microscopy (TEM) results, these authors suggested that the HEX to LAM transition occurs via lateral coalescence of cylinders, implying a perforated or connected lamellar intermediate structure. Unfortunately, the transition they studied is the reverse of that studied here and in general the two may not share the same intermediate state. Also solvent casting used in their work could add additional complications into the transition mechanism. So a direct comparison between their experiment and our theoretical results is difficult to draw. Thermally induced HEX to BCC transition was also studied by Sakurai et al. [47], but the focus there was more to demonstrate the thermal reversibility of this transition than to elucidate the mechanism of the transition.

One of our main results is the prediction that the PL-ML structure serves as an intermediate during the transition from LAM to HEX in certain temperature ranges. We now discuss, in some more detail, the nature and stability of the PL-ML structure. Thomas and co-workers [32] were the first to propose a perforated lamellar (which they term the catenoid-lamellar) structure as a model for a microstructure that appears during the rearrangement of a lamellar morphology in thin films of poly(styrene-b-butadiene) diblocks. The model they proposed is a bicontinuous structure where both the PS and PB components are three dimensionally connected. Bates and co-workers, using SANS and TEM techinques, have demonstrated the existence of a bulk perforated lamellar structure in the weak-(to-intermediate) segregation regime in both poly(ethylene-propylene-bethylethylene) and poly(styrene-b-isoprene) diblock

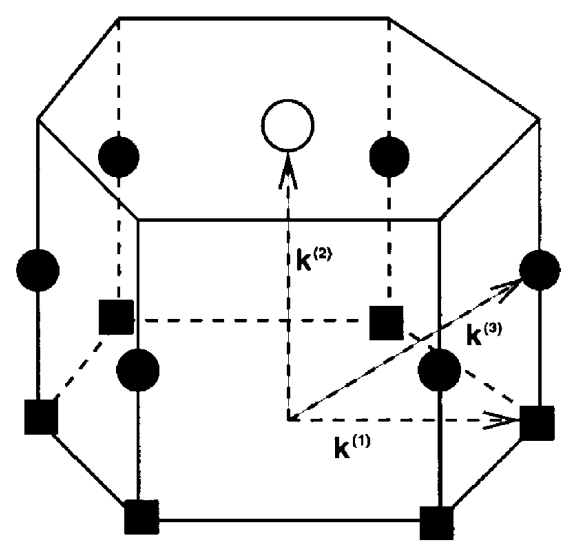

FIG. 16. The first three groups of wave vectors of a hcp structure.

copolymers [31] [Based on rheological measurements, these authors further distinguished between a hexagonally modulated lamellar (HML) at the lower temperature and a hexagonally perforated lamellar (HPL) at a higher temperature. However, the two structures do not seem to have different symmetry; their difference seem to be simply a matter of the degree of the in-plane density modulation.] The structure they proposed is monocontinuous, where the perforations within the lamellar layers have sixfold symmetry, and where the perforations between adjacent layers are staggered. Several calculations $[48,49]$ have addressed the thermodynamic stability of this structure, however, they fail to capture some of the essential structural features which are now believed to be associated with the ML-PL structure $[31,33,29,50]$. More recent state-of-the-art self-consistent field calculations [28,29] using a hexagonal-close-packed (hcp) lattice [51] representation of the PL-ML phases have conclusively shown that within mean-field theory, these structures are not the lowest free energy states. Hamley and Bates [50] had reached the same conclusion earlier based on the hop presentation by keeping modes corresponding to the first two lowest wave numbers.

On the other hand, the structural nature of the PL-ML phase has not been fully resolved. Hamley et al. [50, 31] suggested that PL-ML structure is analagous to the hexatic phase in liquid crystals [52] which consists of weakly coupled layers, with in-plane hexagonal bond-orientational order. This suggestion is based in part on the unusually weak sixfold scattering pattern (and lack of higher harmonics) in the $q_{z}=0$ plane of the $q$ space (where $z$ is the layer normal of the lamellae). Here we offer an alternative proposal. We believe that the PL-ML structure can be represented by an underlying hcp lattice. The first three lowest groups of wave vectors are shown in Fig. 16. These are the same set of wave vectors conceived by Hamley and Bates [50]. However, these authors took the first group of wave vectors to correspond to the optimal wave number $k^{*}$ and only included harmonic corrections due to the second group. We propose that the density waves are dominated rather by the second and third groups whose magnitude, rather than that of the first, are closest to the optimal wave number. Assuming further that $k^{(2)}=k^{(3)}=k^{*}$, we find a stationary structure with $A^{(2)} \neq 0, \quad A^{(3)} \neq 0 \quad$ and $\quad\left|A^{(2)}\right| \gg\left|A^{(3)}\right| \gg\left|A^{(1)}\right|$. A threedimensional gray-level plot is shown in Fig. 17. Except for 


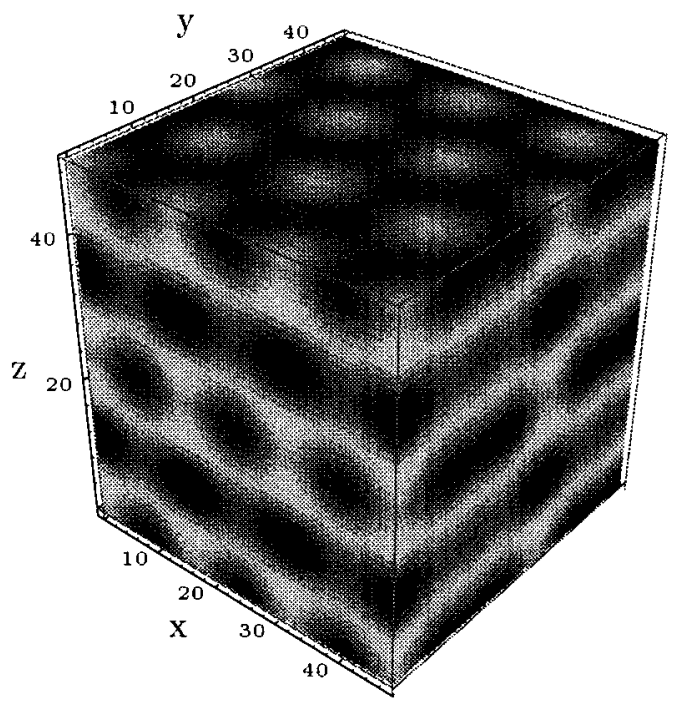

FIG. 17. The pseudostable PL structure constructed using the three groups of wave vectors in Fig. $16(f=0.45, \tau=0.25)$.

some minor details, our proposed structure is consistent with the known structural characteristics of the PL-ML phase. In particular, the weak sixfold scattering spots in the $q_{z}=0$ plane is seen to arise from the very small amplitude of $A^{(1)}$. In addition, from Fig. 16, assuming $k^{(2)}=k^{(3)}=k^{*}$, it follows that $k^{(1)}=(\sqrt{3} / 2) k^{*}$, leading to an in-layer period which is 1.15 times the interlayer spacing; this compares well with the factor 1.1 deduced by Hamley et al. [31] from the small-angle neutron scattering (SANS) data. Furthermore, the ratio $\left|A^{(3)} / A^{(2)}\right|$ is found to increase with increasing temperature, again in agreement with the observations of the same group. Calculation of the stability matrix shows that in the weak-segregation limit (characterized by structures that are dominated by a single, optimal wave number), the PL structure is always a saddle point where a stationary point exists and hence is only pseudostable. In the intermediate-segregation regime, the PL structure can become metastable with positive local curvatures in the free energy but does not have the lowest free energy. The ML structure is either a PL with weak in-layer density modulations, or is the transient state via which the pseudostable LAM state decays.

Recently, Yeung et al. [53] studied the anisotropic fluctuations about the lamellar phase. By performing a linear stability analysis, these authors find that upon increasing temperature, the lamellar phase becomes unstable with respect to fluctuations with wave vectors that form a ring with $k_{z}=1 / 2 k^{*}$, and $|k|=k^{*}$ in $k$ space. Note that both $k^{(3)}$ in Fig. 16 and the wave vectors associated with the amplitudes $B$ and $C$ in Sec. III lie on this ring. Therefore, we conclude that the ML-PL structure are formed from the anisotropic fluctuations of the lamellar phase which first become unstable with respect to these wave vectors.

Based on this analysis, we suggest two possibilities concerning the PL structure observed in experiments. One possibility is that these structures are indeed kinetic, intermediate states en route from the LAM to HEX and other higher temperature phases. Results from more recent experimental studies seem to be leaning toward such a possibility [54].
Another possibility is that thermal fluctuation or other symmetry-breaking mechanisms (such as shear) can lead to stabilization of a pseudostable or metastable state. We are currently exploring these possibilities.

Thermally induced LAM to HEX transition has been studied by Hajduk et al. [55] for the poly(styrene-b-ethene-cobutene) diblock copolymer. However, these authors did not observe the appearance of the intermediate PL-ML structure. We suspect that the thermal annealing used in this study and the long intervals between observations is responsible for missing this intermediate structure: since the PL structure is a saddle point in the free energy, its lifetime is shortened by thermal fluctuations associated with the annealing, making the pseudostable structure to disappear at an early stage. It is also possible that the $T$ jump is too large for the PL state to appear (cf. Fig. 15).

As regards other transitions, Bates and co-workers have studied the HEX to BCC [39] and HEX to DIS transitions [56] for a series of poly(ethylenepropylene-b-ethylethylene). These authors showed that BCC spheres pinch off from the HEX cylinders such that the cylinder axis is the (111) direction of the resulting BCC structure which they term the "epitaxial" relationship. They also suggested that the BCC phase form by way of an undulating cylinder structure. These are in agreement with our observed mechanism; see Fig. 7(b). More interestingly, these authors reported observation of a transient sphere structure during the melting of the HEX in shear cessation experiments. In one of these experiments, an initially disordered phase of asymmetric PEP-PEE close to the order-disorder boundary is subjected to a constant shear which induces a transition to the HEX phase (with the cylinders aligned along the shear direction). The shear is suddenly stopped, and the system is now in a condition favoring the DIS phase. These authors observed that the cylinders first break into spheres before melting to the DIS phase, much akin to what we find after a temperature jump. Indeed insofar as a HEX phase is created and then the condition is changed to favoring the DIS phase, the shearcessasion experiment can be likened to a temperature jump experiment. We believe the mechanism described in this paper is responsible for their observations.

In conclusion, on the basis of numerical simulation and mode analysis in the framework of the TDGL approach, we have provided a comprehensive picture of the different mechanisms in the order-order and order-disorder transitions of block copolymer and other weakly segregated microstructured systems. The inclusion of different kinetic scenarios considerably broadens the phase diagram for these systems. We hope that our results will provide important insights to experiments that are directly or indirectly related to microphase transition kinetics in block copolymers and other related systems.

\section{ACKNOWLEDGMENTS}

This research is supported in part by the National Science Foundation (Grant Nos. ASC-9217368 and DMR-9531914) and the Camille and Henry Dreyfus Foundation (Award No. TC-96-063). 
[1] See F.S. Bates, Science 251, 898 (1991); F.S. Bates and G.H. Fredrickson, Annu. Rev. Phys. Chem. 41, 525 (1990); G.H. Fredrickson and F.S. Bates, Annu. Rev. Mater. Sci. 26, 501 (1996).

[2] S.Y. Qi and Z.-G. Wang (unpublished).

[3] J.D. Gunton, M.S. Miguel, and P.S. Sahni, Phase Transition and Critical Phenomena, edited by C. Domb and J.L. Lebowitz (Academic, New York, 1983), Vol. 8.

[4] F.H. Stillinger, J. Chem. Phys. 78, 4654 (1982).

[5] T. Ohta and K. Kawasaki, Macromolecules 19, 2621 (1986).

[6] D. Wu, D. Chandler, and B. Smit, J. Chem. Phys. 96, 4077 (1992); M.W. Deem and D. Chandler, Phys. Rev. E 49, (1994); 49, 4268 (1994).

[7] L. Leibler, Macromolecules 13, 1602 (1980).

[8] A.M. Mayes, M.O. de la Cruz, and W.E. McMullen, Macromolecules 26, 4050 (1993).

[9] M. Seul and D. Andelman, Science 267, 476 (1995), and references therein.

[10] K. Kawasaki and K. Sekimoto, Physica A 143, 349 (1988); Physica A 148, 361 (1988).

[11] V. Kumaran and G.H. Fredrickson, Physica A 204, 378 (1994).

[12] See, also, M. Doi and S.F. Edwards, The Theory of Polymer Dynamics (Clarendons, Oxford, 1986).

[13] C. Roland and R.C. Desai, Phys. Rev. B 42, 6658 (1990); C. Sagui and R.C. Desai, Phys. Rev. Lett. 71, 3995 (1993); Phys. Rev. E 49, 2225 (1994); 52, 2807 (1995); 52, 2822 (1995); Phys. Rev. Lett. 74, 1119 (1995).

[14] S. Puri and Y. Oono, Phys. Rev. A 38, 1542 (1988).

[15] Y. Oono and Y. Shiwa, Mod. Phys. Lett. B 1, 49 (1987); M. Bahiana and Y. Oono, Phys. Rev. A 41, 6763 (1990).

[16] A. Chakrabarti, R. Toral, and J.D. Gunton, Phys. Rev. E 47, 3025 (1993).

[17] G.H. Fredrickson and R.G. Larson, J. Chem. Phys. 86, 1553 (1987); R.G. Larson and G.H. Fredrickson, Macromolecules 20, 1897 (1987).

[18] G.H. Fredrickson and E. Helfand, J. Chem. Phys. 89, 5890 (1988).

[19] K. Kawasaki and A. Onuki, Phys. Rev. A 42, 3664 (1990).

[20] M. Rubinstein and S.P. Obukhov, Macromolecules 26, 1740 (1993)

[21] A. Onuki, J. Chem. Phys. 87, 3692 (1987).

[22] M.E. Cates and S.T. Milner, Phys. Rev. Lett. 62, 1856 (1989).

[23] G.H. Fredrickson, J. Rheol. 38, 1045 (1994).

[24] T. Ohta, Y. Enomoto, J.L. Harden, and M. Doi, Macromolecules 26, 4928 (1993); 26, 4935 (1993); H. Kodama and M. Doi, ibid. 29, 2652 (1996).

[25] For an excellent review of theoretical and experimental work on the dynamics of block copolymers, see G.H. Fredrickson and F.S. Bates [1] (the third paper).

[26] S.Y. Qi and Z.-G. Wang, Phys. Rev. Lett. 76, 1679 (1996).

[27] D.A. Hadjuk, P.E. Harper, S.M. Gruner, C.C. Honecker, G. Kim, E.L. Thomas, and L.J. Fetters, Macromolecules 27, 4063 (1994).

[28] M.W. Matsen and M. Schick, Phys. Rev. Lett. 72, 2660 (1994); Macromolecules 27, 4014 (1994).

[29] M.W. Matsen and F.S. Bates, Macromolecules 29, 1091 (1996).

[30] R. Kikuchi, T. Mohri, and B. Fultz, MRS Symposium Pro- ceedings 205, 387 (1992); B. Fultz, Philos. Mag. B 67, 253 (1993).

[31] I.W. Hamley, K.A. Koppi, J.H. Rosedale, F.S. Bates, K. Almdal, and K. Mortensen, Macromolecules 26, 5959 (1993); S. Förster, A.K. Khandpur, J. Zhao, F.S. Bates, I.W. Hamley, A.J. Ryan, and W. Bras, ibid. 27, 6922 (1994).

[32] E.L. Thomas, D.M. Anderson, C.S. Henkee, and D. Hoffman, Nature 334, 598 (1988).

[33] M.M. Disco, K.S. Liang, S.K. Behal, R.J. Roe, and K.J. Jeon, Macromolecules 26, 2983 (1993).

[34] The time unit we use in this paper is $d^{2} /\left(200 M k_{B} T\right)$, where $d$ is the period of the microstructure corresponding to the optimal wave number, $M$ is the mobility coefficient, and $\left(M K_{B} T\right)$ can be thought of as the collective, mutual diffusion coefficent.

[35] In general it is desirable to be in the more asymmetric part of the phase diagram since the windows for the different phases get wider. In the temperature jumps involving the lamellar phase, however, increasing the asymmetry also increases the temperature difference between the different phases and too large a temperature difference can sometimes lead to numerical instabilities in the simulation. This is the reason why $f=0.45$ was used in the cases involving the lamellar phase.

[36] M.C. Cross and P.C. Hohenberg, Rev. Mod. Phys. 65, 851 (1993).

[37] The dominant wave vector determined from the dynamic equation is slightly different from that determined from the free energy. However, consistent with the single wave length approximation, we ignore this difference.

[38] The sign of $A_{n} \mathrm{~s}$ are determined by the miniminization of the free energy and there are several equivalent choices. In this paper, we shall choose $A_{1}$ and $A_{4}$ to be negative, while all the other $A_{n} \mathrm{~s}$ are positive.

[39] M.F. Schulz, F.S. Bates, K. Almdal, and K. Mortensen, Phys. Rev. Lett. 73, 86 (1994); K.A. Koppi, M. Tirrell, F.S. Bates, K. Almdal, and K. Mortensen, J. Rheol. 38, 999 (1994).

[40] S. Qi and Z.-G. Wang (unpublished).

[41] S.A. Brazovskii, Zh. Éksp. Teor. Fiz. 68, 175 (1975) [Sov. Phys. JETP 41, 85 (1975)].

[42] G.H. Fredrickson and E. Helfand, J. Chem. Phys. 87, 697 (1987).

[43] M. Muthukumar, Macromolecules 26, 5259 (1993).

[44] T. Hashimoto, K. Kowsaka, M. Shibayama, and S. Suehiro, Macromolecules 19, 750 (1986); T. Hashimoto, K. Kowsaka, M. Shibayama, and H. Kawai, ibid. 19, 754 (1986).

[45] M.A. Singh, C.R. Harkless, S.E. Nagler, R.F. Shannon, and S.S. Ghosh, Phys. Rev. B 47, 8425 (1993); C.R. Harkless, M. A. Singh, S.E. Nagler, G.B. Stepheson, and J.L. Jordan-Sweet, Phys. Rev. Lett. 64,2285 (1990).

[46] S. Sakurai, T. Momii, K. Taie, M. Shibayama, S. Nomura, and T. Hashimoto, Macromolecules 26, 485 (1993).

[47] S. Sakurai, H. Kawada, T. Hashimoto, and L.J. Fetters, Macromolecules 26, 5796 (1993).

[48] G.H. Fredrickson, Macromolecules 24, 3456 (1991).

[49] M. Olvera de la Cruz, A.M. Mayes, and B.W. Swift, Macromolecules 25, 944 (1992).

[50] I.W. Hamley and F.S. Bates, J. Chem. Phys 100, 6813 (1994).

[51] The term hcp is used even though the actual $c / a$ ratio differs from that corresponding to the closest packing $c / a=\sqrt{3}$; see C. Kittel, Solid State Physics (Wiley, New York, 1976). 
[52] P.S. Pershan, Structure of Liquid Crystals (World Scientific, Singapore, 1988).

[53] C. Yeung, A.-C Shi, J. Noolandi, and R.C. Dasai, Macromol. Theory Simul. 5, 291 (1996).

[54] D.A. Hajduk, H. T. Takenouchi, M. A. Hillmyer, F. S. Bates, M. E. Vigild, and K. Almdal (unpublished).

[55] D.A. Hajduk, S.M. Gruner, P. Rangarajan, R.A. Register, L.J.
Fetters, C. Honeker, R.J. Albalak, and E.L. Thomas, Macromolecules 27, 490 (1994).

[56] K.A. Koppi, Ph.D. thesis, The University of Minnesota, 1994 (unpublished); F.S. Bates, K.A. Koppi and M. Tirrell, Macromolecules 27, 5934 (1994); K. Almdal, K. Mortensen, K.A. Koppi, M. Tirrell, and F.S. Bates, J. Phys. (France) II 6, 617 (1996). 